\title{
Rehabilitation, Quality of Life and Survival
}

\section{Survival Analysis of Peritoneal Dialysis Patients under PD First Policy of Thailand}

Introduction and Aims: PD first policy was implemented in Thailand since January 2008 by National Health Security Office (NHSO). The study was conducted to evaluate patient survival and its associated factors of patients who performed PD under the policy.

Method: The records of PD patients from the database of NHSO during October 2007-February 2012 were analyzed. Patients who survived at least 30 days after the first treatment were recruited to the study. Age and gender of patients were analyzed by univariate analysis. Causes of death were categorized as follows: non-PD related infection, PD-related infection, cardiovascular-associated cause, malnutrition, social problem-related cause and miscellaneous

Results: Total 15,511 cases were registered to the policy, the data of 14,609 patients who fit to the criteria were analyzed. The median age was 57 years old Male to female ratio was close to $1: 1$. At the end of study period, $70.2 \%$ of the patients were alive. The 1-, 2-, 3- and 4-year patient survival rates were $79.03 \%$ (95\%Cl:78.30-79.74\%), 65.77\% (95\% Cl: 64.78-66.73\%), 56.82\% $(95 \% \mathrm{Cl}$ $55.5-58.12 \%$ ), $49.95 \%$ (95\% Cl:47.61-52.25\%), respectively. Higher age was significantly associated with lower patient survival (see table, $p<0.05^{\star}$ ). Major known causes of death in 1,381 cases were non-PD related infection $(28.96 \%)$, cardiovascular-related cause (26.43\%), and PD-related infection (11.08\%).

\begin{tabular}{ccc} 
Age (years old) & \multicolumn{3}{c}{ Hazard ratio $(95 \%$ Cl) } \\
\hline $25-34$ & 1.0 & $(0.81-1.29)$ \\
$35-44$ & 1.0 & $(0.82-1.25)$ \\
$45-54$ & 1.5 & $(1.23-1.81)^{\star}$ \\
$55-64$ & 2.0 & $(1.66-2.41)^{\star}$ \\
$65-74$ & 2.6 & $(2.14-3.14)^{\star}$ \\
$\geq 75$ & 4.2 & $(3.38-5.14)^{\star}$
\end{tabular}

Conclusions: The patient survival of PD patients under the PD first policy is acceptable. Age of patients is associated with the survival. The main known causes of death are non-PD related infection and cardiovascular-related cause.

Changsirikulchai S., ${ }^{1}$ Ariyapitipan S., ${ }^{2}$ Cheungsaman P., ${ }^{3}$ Sirivongs D. ${ }^{4}$ Srinakharinwirot University, ${ }^{1}$ Bangkok; National Health Security Office; ${ }^{2}$ Banphaeo Hospital (Public Organization), ${ }^{3}$ Bangkok; Khon Kaen University, ${ }^{4}$ Khon Kaen, Thailand.

\section{Quality of Life in Different Modalities of Peritoneal Dialysis}

Introduction: Ambulatory peritoneal dialysis (APD) is perceived to provide a better quality of life (QoL) than continuous ambulatory peritoneal dialysis (CAPD).

Objectives: To compare the QoL between APD and CAPD patients and factors affecting it.

Methods: QoL was evaluated using the Medical Outcomes Study 36-item Short Form Health Survey questionnaire in English and Malay in PD patients more than 4 months duration.

Results: 50 patients [20 male (40\%), 30 female (60\%)] were recruited. Majority were married (58\%) and had secondary education (54\%), but only $32 \%$ were still in full-time employment. The table shows the scores on the QoL. Age correlated with physical function ( $p=0.006, r=-0.381$ ) and physical component summary $(p=0.021, r=-0.326)$. There was a trend towards linear relationship between physical function score with $\mathrm{Kt} / \mathrm{V}(p=0.059$ and $\mathrm{r}=0.269)$.

\begin{tabular}{lccc} 
& CAPD & APD $(n=10)$ & $P$ \\
& $(n=40)$ & & value \\
\hline Age (years) & $49.05 \pm 14.92$ & $38.60 \pm 17.15$ & 0.06 \\
Duration ( months) & $37.57 \pm 27.26$ & $11.23 \pm 10.87$ & 0.005 \\
$\mathrm{Hb}($ g/dl) & $10.79 \pm 1.28$ & $12.23 \pm 2.08$ & 0.008 \\
$\mathrm{Kt} / \mathrm{v}$ & $2.12 \pm 0.32$ & $1.99 \pm 0.64$ & 0.37 \\
Albumin ( g/L) & $37.97 \pm 4.55$ & $37.8 \pm 4.02$ & 0.912 \\
Physical function & $54.08 \pm 32.91$ & $64.5 \pm 23.39$ & 0.352 \\
Role physical & $56.87 \pm 44.57$ & $57.5 \pm 37.36$ & 0.968 \\
Bodily pain & $67.05 \pm 23.42$ & $63.1 \pm 17.34$ & 0.62 \\
General health & $57.72 \pm 18.73$ & $52.40 \pm 19.15$ & 0.427 \\
Vitality & $63.0 \pm 19.6$ & $59.5 \pm 22.54$ & 0.626 \\
Social function & $70.93 \pm 25.69$ & $66.25 \pm 23.6$ & 0.603 \\
Role emotional & $65.83 \pm 42.36$ & $70.01 \pm 39.9$ & 0.779 \\
Mental health & $72.37 \pm 17.7$ & $67.6 \pm 18.61$ & 0.455 \\
Physical component summary (PCS) & $40.13 \pm 9.96$ & $41.28 \pm 7.07$ & 0.735 \\
Mental component summary (MCS) & $50.43 \pm 10.17$ & $47.83 \pm 10.12$ & 0.474
\end{tabular}

Conclusion: APD patients had a comparable QoL with CAPD patients despite the younger age, shorter duration, and higher hemoglobin.

Mohd R., Cader R.A., Ibrahim S., Abd Rahman W.K., Haslina Hassan W., Rosman N.A.S., Gafor H., Juliaty E., Kong W.Y., Kong N.C.T. Universiti Kebangsaan Malaysia, Cheras, Bandar Tun Razak, Kuala Lumpur, Malaysia.

\section{Assisted APD Using Paid Community Health-care Assistants: UK Single Center Experience}

Introduction: The elderly are often considered to be ineligible for PD. Assisted automated peritoneal dialysis (aAPD) is being developed in the UK to broaden access to PD. It is primarily intended for the frail and elderly but can also be used for younger patients unable to perform self-care PD but who wish to dialyse at home.

Aim: Retrospective study of the aAPD program at a single center implemented from 2007 following a successful pilot project in 2005.

Method: A third-party health-care agency is used to provide community health-care assistants ( $\mathrm{HCA}$ ) trained and supervised by Baxter Healthcare (UK). The assistance is once a day for a 30-45 minute visit. The HCA dismantles the overnight APD equipment and re-assembles the cycler for use at night. Additional assistance for some patients includes weight, blood pressure measurement and PD exit site dressing. Patient selection: Ability to safely connect/disconnect from cycler is essential. All have $\geq 2$ comorbidity scores and barriers to self-care PD.

Results: 12 (9 female, 3 male) patients registered. Mean age starting aAPD 67.5 (32-82) years. Origin of care: 2 hemodialysis, 4 self-care PD, 6 predialysis. 3 patients had shared aAPD (family/personal carer). Outcomes: Length of time on assistance: 29 days-24 months to date. Reason for stopping: 1 ultrafiltration failure and transferred to hemodialysis, 3 deaths, 3 transferred to independent self-care PD. Only 1 patient (on shared aAPD) has had peritonitis; this was due to pseudomonas and was concurrent with PD exit-site infection.

Conclusion: aAPD using HCAs is an affordable dialysis modality within the UK NHS (National Health Service). Such assistance enables patients to stay on PD when unable to continue independently and provides a homebased treatment for patients too frail to carry out PD entirely themselves. Of note, some do become independent after time on aAPD.

Haddoub S.S., Clemenger M., McGrory J., Hisole N., Brown E.A. West London Renal and Transplant Centre, Imperial College Healthcare NHS Trust, London, United Kingdom.

\section{Erectile Dysfunction in Men on Continuous Ambulatory Peritoneal Dialysis}

Background: Erectile dysfunction (ED) is sexual dysfunction characterized by the inability to develop or maintain an erection of the penis during sexual performance. Several somatic and psychological mechanisms have been implicated in the etiology of sexual dysfunction. Sexual dysfunctions consist of erectile dysfunction and decreased libido or loss of sexual interest. A number of previous studies have documented the associated of age, disease and health behaviors with erectile dysfunction. Patients with chronic kidney disease have higher risks for ED. The aim of study was to access the prevalence of erectile dysfunction in men on continuous ambulatory peritoneal dialysis (CAPD) in Cipto Magunkusumo Hospital.

Methods: Descriptive observational study. The range of age is between 30-60 y/0. They were divided into 3 different age groups: $<40 \mathrm{y} / 0,40-50 \mathrm{y} / 0$ and $>50 \mathrm{y} / 0$. We used international index of erectile dysfunction questionnaire 5 (IIEF5) as a diagnostic tool for erectile dysfunction

Results: There were 21 patients enrolled in this study. Patients have been CAPD $>24$ months. Mild erectile dysfunction $24 \%$, moderate $14 \%$ and severe $43 \%$.

Conclusion: Sexual dysfunction is common among patients with chronic kidney disease. Prevalence of ED $81 \%$ in CAPD patients at Cipto Mangunkusumo Hospital.
Harnavi H. Division of Kidney, Department Internal Medicine of Cipto Mangunkusumo Hospital, Jakarta, Indonesia. 


\section{Rehabilitation, Quality of Life and Survival}

Ultrabag Connecting Device: a Connection Device in Empowering Marginal Patients on Self-performing CAPD

Objectives: CAPD is the most prevalent initial form of RRT in Hong Kong. The empowerment of marginal patients with specially designed device can result in safe CAPD connections.

Method: From 2007-2010, 59 marginal patients where PD bags connections were otherwise considered unsafe in the past were trained on self-care CAPD with a Ultrabag Connecting (UC) device. 44 of them were new patients and their outcomes were compared with a control group of 65 patient.

Results: There were no significant difference in mean age at CAPD training $(p=0.433)$ and in sex ratio $(p=0.31)$ between the 2 groups. The proportion of diabetic was $40 / 44$ in the device group and was significantly higher than the control group $(p=0.000)$. All patients using device performed PD exchanges by themselves. The reasons for the use of the device were: poor eye sight $(n=34)$, mono-ocular vision $(n=1)$ and hand tremor $(n=9)$. The degree of impaired visual acuity ranged from (20/100) to hand movements only. Average training period was 6.7 days and 6.6 days, respectively $(p=0.932)$. For a cumulative treatment period of 1143 patient-months in the device group, the peritonitis rate was 1 in 45.7 patient-months and the corresponding rate for control group was 1 in 39.2 patient-months $(p=0.000)$.

Conclusion: In this observational study, the UC device empowered our visually impaired or hand tremor CAPD patients to take care of themselves, and the empowerment process resulted in superior performance.

Ho Y.W. ${ }^{1}$, Lam W.O. ${ }^{1}$, Cheng Y.Y. ${ }^{1}$, Tang W.C. ${ }^{1}$, Wong S. ${ }^{1}$, Ho E.T.L. ${ }^{1}$, Wan, $\mathrm{M}^{2}$. Renal Unit, Department of Medicine, ${ }^{1}$ and Occupational Therapy Department, ${ }^{2}$ United Christian Hospital, Hong Kong, China.

\section{Prevalence and Risk Factors of Uraemic Pruritus in CAPD Patients}

Objectives: Uraemic pruritus is a distressing symptom which has a negative impact on quality of life (QoL) of the patients. In the present study, we aimed to assess the prevalence and related risk factors of uraemic pruritus among continuous ambulatory peritoneal dialysis (CAPD) patients.

Methods: The demographic information, clinical data and laboratory findings in CAPD patients in our PD center from January, 2009 to April, 2010 were collected. Pruritus was assessed by a self-reported questionnaire in CAPD patients. Patients' sleep quality, depression, and QOL were assessed using the Pittsburgh Sleep Quality Index (PSQI), Beck Depression Inventory (BDI), and Short Form 36 (SF-36), respectively.

Results: Totally 362 CAPD patients were investigated. Pruritus was observed in $65.6 \%$ percent CAPD patients. Compared to patients without pruritus, patients with pruritus had higher diabetes mellitus proportion ( $31.4 \%$ vs. $15.5 \%, p=0.044)$, poorer sleep quality $(10.3 \pm 4.8$ vs. $7.7 \pm 5.3$, $p=0.001)$, longer duration of CAPD ( $26.9 \pm 2.2$ vs. $16.6 \pm 1.5, p=0.001)$, higher calcium phosphorous product levels ( $52.1 \pm 12.2$ vs. $47.2 \pm 13.8, p=0.005$ ), and higher Intact-PTH level $(426.9 \pm 38.1$ vs. $271.6 \pm 26.5, p=0.01)$. The correlation analysis demonstrated that pruritus was positively correlated with sleep quality $(r=0.24, p=0.001)$, CAPD vintage $(r=0.23, p=0.001)$, diabetes mellitus ( $r=0.15, p=0.034$ ), calcium phosphorous product levels $(r=0.15, p=0.035)$ and Intact-PTH level $(r=0.19, p=0.01)$, while negatively correlated with SF-36 physical component score (PCS) $(r=-0.19, p=0.007)$ Using multiple logistic regression analysis, we found that Intact-PTH level $(\mathrm{OR}=1.52, p=0.004)$, and CAPD vintage $(\mathrm{OR}=1.04, p=0.004)$ were independently associated with pruritus.

Conclusions: Pruritus was independently associated with longer PD vintage and higher intact-PTH level. Our results suggest that control of intact-PTH level may be beneficial to uraemic pruritus in CAPD patients.

Jianying Li, Qunying Guo, Jianxiong Lin, Xiaoging Ye, Chunyan Yi, Xiao Yang, Xueqing Yu Department of Nephrology, The First Affiliated Hospital, Sun Yat-sen University, Guangzhou, China.

\section{Outcome of Patients on CAPD in Bangladesh: Comparative Reports from Two Different Time Periods in the Last Decade}

Objectives: CAPD is an alternate modality of dialysis that is less practiced than hemodialysis worldwide. In Bangladesh penetration of PD is also low. It has been restarted some fifteen years back and growing continuously since then. In this paper we reviewed the outcome of PD over two different time periods in the last decade.

Methods: The two time periods are for group 1 (early: 1995-2000) and group 2 (later: 2001-2008). Around 478 patients took PD being 180 vs. 298 in the two periods. In all patients, a Tenckhoff double-cuff, catheter (mostly straight) was inserted surgically. In group 1, patients used various types of PD connections like straight tubing (spike or a luer lock device) or $Y$ connection systems, and group 2 used mostly $Y$ connection or double-bag (twin bag) systems.

Results: Available data showed mean age of group 1 and 2 subjects respectively was $61 \pm 7$ vs. $57 \pm 18$ years, $(p=0.09$ ); male and female ratio $61 \%$ and $39 \%$ vs. $56 \%$ and $14 \%,(p=0.47)$ and primary renal diseases were $D N$ and $G N$ as $54 \%$ and $20 \%$ vs. $64 \%$ and $18 \%,(p=0.06)$. Indication for PD in two groups were hemodynamic instability, patient's choice, $\mathrm{HD}$ unavailability and access failure as $51 \%, 22 \%, 22 \%$ and $5 \%$ vs. $57 \%, 16 \%, 14 \%$ and $13 \%$, ( $p=0.03$ ). Rate of peritonitis in both groups was $1 / 9$ vs. $1 / 21$ patient-months and exitsite infection $1 / 26$ vs. $1 / 93$ patient-months. Technique survival was $89 \%$ vs. $98 \%$ at 1 year $(p=0.01)$. Patient survival was $83 \%, 52 \%$ and $48 \%$ and $64 \%, 46 \%$ and $32 \%,(p=0.45)$ at 1st, 2nd and 3rd year, respectively. The most frequent causes of deaths were cerebrovascular accident, cardiac complications, and sepsis in both groups.

Conclusions: It may be concluded that the trend in PD is still for elderly diabetics who are mostly unsuitable for hemodialysis. Over the period, possibly with the use of newer connection systems, the incidence of peritonitis and exit infection has significantly come down. Patient survival and pattern of death have remained similar. CAPD can be a preferred alternate to these compromised patients.

Iqbal M.M.,${ }^{1}$ Iqbal S., ${ }^{2}$ Samad M.A., ${ }^{3}$ Islam M.N., ${ }^{4}$ Rahman M.H.,${ }^{5}$ Rashid H.U. ${ }^{5}$ Nikdu Hospital, ${ }^{1}$ Birdem Hospital, ${ }^{2}$ Lab Aid Specialized Hospital, ${ }^{3}$ UHL Hospital, ${ }^{4}$ and BSM Medical University, ${ }^{5}$ Dhaka, Bangladesh.
Thailand Key Performance Indicators (KPIs) for Peritoneal Dialysis

Objective: To ensure quality of nationwide PD service paralleling the burst of PD cases in Thailand, survey of key performance indicators (KPIs) was performed.

Methods: All PD centers in Thailand were invited to participate in the present study. PD nurse case managers in each center were asked to review medical records of all patients undergoing PD during October, 2009 to September, 2010 and submitted data to the main investigators.

Results: About three-fourths of all PD-centers in Thailand (86 out of 121) participated in the present study. 105 nephrologists and 154 PD nurse specialists served 7,339 PD (279 APD and 7,060 CAPD) patients in these centers. Overall annual dropout rate was $24.8 \%$ and mortality rate was $17.1 \%$. Nephrologist/patient ratio was $1: 58$, and certified PD nurse/ patient (N/P) ratio was $1: 85$. Of these, $33.7 \%$ had $N / P$ ratio $>1: 60$ and $41.9 \%$ had no nephrologist in their center. Overall exit-site infection rate was 1 episode/40.7 pt-mo ( 0.30 episodes/pt-yr), while overall peritonitis rate was 1 episode/25.8 pt-mo (0.47 episode/pt-yr). Of interest, larger (>100 cases), community-based, PD nurse-overloading, nonnephrologist coverage centers had higher peritonitis. Culture negative was $32.1 \%$. Almost patients $(90.4 \%)$ received predialysis education and the home visit rate was $37.8 \%$.

Conclusion: Despite of the rapid growth of PD cases under the limited resource, the PD-related infection rates in Thailand are only small degree behind the goal of Asia-Pacific Key Performance Indicators (KPIs) Task Force. 


\section{Rehabilitation, Quality of Life and Survival}

\section{Multidisciplinary Care Program for Patients on Long-term Peritoneal Dialysis}

Uncertainty, frustration, stress and anxiety are the kind of experiences patients with renal failure. Predialysis care could improve their performance, psychosocial well being and quality of life (QOL) through a multidisciplinary approach provided by the medical and allied health groups. We study the efficacy of this program in improving patients' QOL and psychosocial status. All consecutive patients started on long-term dialysis during the period March to Dec 2011 were included. Each had two QOL assessments using the Kidney Disease and Quality of Life (KDQOL-SF) and HOQOL-questionnaires. Anxiety and depression were assessed by Hospital Anxiety and Depression Scale (HADS). Patients' satisfaction was rated by Occupational Performance Appraisal Questionnaire (OPAQ) which contains 10 questions reviewing self satisfaction towards daily life and health status in the recent 2 weeks. 36 patients (22 female and 14 male, age $55.5+10.3 \mathrm{yr}$ ) were recruited. WHOQOL-BREF showed low scores on psychological health (12.81) and social relationships (13.75), while the physical (14.43) and environment (14.69) domain scores were higher. The anxiety and depression domains of HADS scored 1.88 and 8.1 , respectively. 13 patients with depression scores above 8 were seen by clinical psychologist. For KDQOL, patients also attained higher scores in symptoms (82.7 \pm 15$)$, effect of kidney disease $(76.1 \pm 22)$ and cognitive function $(75.8 \pm 28)$. The scores were lower in burden of kidney $(23.9 \pm 21)$ and social interaction $(69.0 \pm 23)$, suggesting that the chronic kidney disease had a major impact on their lives and QOL. The OPAQ scores (1.27-1.5) showed that the patients were generally satisfied with this new program. To conclude, our patients can benefit from this multidisciplinary program.

Lam M.F. ${ }^{1}$ Lee M.S.C. ${ }^{2}$ Law C.W. ${ }^{3}$ Leung S.Y.H., ${ }^{1}$ Chu M.L.M., ${ }^{2}$ Chan T.M..${ }^{1}$ Department of Medicine, ${ }^{1}$ Queen Mary Hospital, and Allied health Group, ${ }^{2}$ Queen Mary Hospital, and Department of Psychiatry, ${ }^{3}$ Queen Mary Hospital, Hong Kong.

\section{Multidisciplinary Approach to Improving End-of-life Care for Peritoneal Dialysis Patients}

Many aspects of end-of-life care are suboptimal for patients with chronic kidney disease. As part of a collaborative project the peritoneal dialysis program in our center formed a multidisciplinary team to improve care. Identification of PD patients likely to be within the last year of their life was key to the project. A dependency scoring tool already in use to plan nursing input was cross-referenced against objective measures of physical decline, frequency of health-care contacts and subjective feeling of experienced nurses. The MDT discussed each case: a newly piloted tool, "My Wishes", for ascertaining patient's thoughts on future care, was found to be an excellent way of facilitating such difficult discussions. Contact was made with the primary care doctor, placing patients on a "Cause for Concern" register: important in avoiding inappropriate acute hospital admissions for patients near the end of life. Links with palliative care services and local hospices were developed. Patients and their family were invited to a clinic visit with their physician and nurse to discuss prognosis, future care planning, and end-of-life care.

Case study. Deterioration of PD patient noted by specialist nurse in the home. Increased dependency scoring and global decline in 74 year old female on PD for 3 years. Physician made diagnosis of calciphylaxis. MDT review of prognosis and therapeutic alternatives. Home visit by physician and specialist nurse: discussion with patient and family, decision on conservative/palliative care, no change of modality and alterations to medications. Contact made with primary care physician and local palliative care team. Cause for Concern register initiated ensuring optimal support in community and avoidance of hospitals care as family wished. Patient able to prepare for death. "A good death" at home, with symptom control and family support a month later.

Lewis D., Martin J., Knaggs A., Robinson H., Middleton R., Hannay H., Lappin L. Salford Royal NHS Foundation Trust, Salford, United Kingdom.

\section{Quality of Life of Assisted Peritoneal Dialysis Caregivers in} Department of Nephrology, Hospital Kuala Lumpur

Objective: This study intends to measure the QoL of assisted-PD caregivers.

Methods: This cross-sectional study analyzed the QoL of assistedPD caregivers. WHOQOL-BREF questionnaire was used as the tool to measure their QoL.

Results: 24 caregivers answered the questionnaire. Seven $(29 \%)$ of the caregivers were males, 17 females (71\%). Seventeen are Malays (70.8\%), 5 Chinese $(20.8 \%)$ and 2 Indians $(8.3 \%)$. Their average age was 54 years, range between 27-63 years. The average body mass index (BMI) was 27.5 $\mathrm{kg} / \mathrm{m}^{2}$, range $18.0-41.7 \mathrm{~kg} / \mathrm{m}^{2}$. The average duration of assisted-PD was 23.5 months, range 1-84 months. Thirteen (56.5\%) were CAPD caregivers, whereas $11(43.5 \%)$ assisted in automated PD (APD). CAPD caregivers spent an average of 117.5 minutes/day to perform PD, whereas APD caregivers 72.5 minutes/day. There was no significant WHOQOL-BREF score difference between CAPD and APD group ( $p=0.8449)$, male and female $(p=0.386)$, different ethnic groups $(p=0.489)$, age of caregivers $(p=0.131)$, BMI of caregivers ( $p=0.752)$, time spent by caregivers per day $(p=0.817)$ and duration of assisting PD ( $p=0.752)$.

Conclusion: This study did not reveal any direct relationship between QoL of PD caregivers to any of their biodemographic parameters, type, time spent and duration assisting in PD. This is most likely due to small study sample size. We could not exclude that the QoL of PD caregivers is lower than the normal local population due to lack of baseline QoL scores compared to other societies. WHOQOL-BREF was a general scoring system. Specific questions should be used to measure the burden of assisting PD to the QoL of caregivers.

Leong C.M. ${ }^{1}$ Ang J.K., ${ }^{2}$ Supar J., ${ }^{1}$ Bavanandan S. ${ }^{1}$, Department of Nephrology, Hospital Kuala Lumpur, Malaysia

\section{Experience on Dialysis Collaboration Management during Flood Disaster in Thailand: Report from Thai Renal} Flood Relief Task Force

Background: During the major flood disaster (MFD) in Thailand ( Sept.-Dec., 2011), dialysis patients suffered most among chronic disease patients due to continuous need of renal replacement therapy. The Thai Renal Flood Relief Task Force (TRFRTF) was set up in collaboration with The Kidney Foundation of Thailand, The Nephrology Society of Thailand, Thai Transplantation Society, Thai Nephrology Nurse Association, The Government Pharmaceutical Organization, National Healthcare Schemes, and Thai Kidney Patient Club. Its mission was to relieve clinical needs of dialysis patients during MFD.

Objective: To demonstrate our management experience in caring for peritoneal dialysis patients during MFD.

Methods: Working experience, data collection during MFD, and questionnaire sent after MFD were reviewed.

Results: During MFD, 12,499 dialysis patients, 2,000 PD and 10,499 HD, residing in the flood area were affected. TRFRTF was set up in late October and organized the following operation systems including information about flood situation, patient status, logistics of PD fluid and medical supplies, guideline for caring dialysis patients, updated status on dialysis situation and patient information. The information was distributed to nephrologists and dialysis nurses via news, newspapers, email, SMS and Facebook. Regarding PD modality, 10 PD centers were closed and 142 cases were transferred to shelter area elsewhere and were under care of other PD centers. However, $99 \%$ of $P D$ patients had remained in direct contact with $P D$ nurses. Temporary PD stations for supplying PD fluid and medical supplies were set up in the area where patients could get access. Lack of PD fluid, medical supplies and essential drugs were reported in $19 \%, 12 \%$ and $\%$, respectively, of all PD centers. Mortality rate was similar when compared with the normal situation. Due to patients' relocation, an interim reimbursement system was adopted in collaboration with the government health-care schemes. Just after MFD, a workshop was held to improvise readiness on disaster management for the next event (if any).

Conclusion: TRFRTF is very helpful for collaborating and executive crisis management during MFD in Thailand. The success depended heavily on cooperation of all parts involved in clinical services to the Thai dialysis patients.

Lumpaopong A., Chuengsaman P., Bunnag S., Tungsanga K., Sirivongs D., Supaporn T., Aruyapitipan S., Dhanakijcharoen P., Vanichakarn S. On behalf of Thai Renal Flood Relief Task Force, Bangkok, Thailand. 


\section{Rehabilitation, Quality of Life and Survival}

\section{Quality of Life Measurement Based on SF-36 in Patients with ESRD on CAPD in Zainul Abidin General Hospital}

Objective: Morbidity and mortality still take a high place in patients with end-stage renal disease (ESRD) in developing countries, with mortality rate approximately $22 \%$. Continuous ambulatory peritoneal dialysis (CAPD) is one of therapeutic modality for ESRD. The assessment of quality of life is important to measure the effectiveness and adequacy of dialysis therapy. Short form-36 (SF-36) questionnaire also can be used to measure the physical and psychological factors. The aim of this study is to evaluate the quality of life in patient with CAPD based on SF-36.

Methods: This descriptive research which sample collected from Zainu Abidin General Hospital. Total subject 22 men, retrieval data using SF-36 questionnaire directly to the patient.

Results: The majority of patients with CAPD were $40-59$ years old, were 12 patients $(54.55 \%)$. The etiology most causes by diabetes mellitus. More of the patients have a good mental health score $(77.27 \%)$, compared with a good physical health's score $(72.27 \%)$. The numbers with good quality of life were $19(86.36 \%)$ and the poor were $3(13.64 \%)$.

Conclusion: This study shows that patients with CAPD have a good quality of life but less in general health. Need to give more motivation in order to have better general health.

Maimun . $_{.,}{ }^{1}$ Ibrahim P., ${ }^{2}$ Shinta A. ${ }^{3}$ Division of Nephrology of Internal Medicine Department, ${ }^{1}$ Psychiatry Department, ${ }^{2}$ Syiah Kuala University Faculty of Medicine, ${ }^{3}$ Banda Aceh, Indonesia.

\section{Survival Rate in ESRD Patients on CAPD in Zainul Abidin General Hospital Correlation with Age and Etiology}

Objective: End-stage renal disease (ESRD) is one of the most common worldwide diseases which the incidence and prevalence is increasing each year. Renal replacement therapy (RRT), indeed, in order to survive, continuous ambulatory peritoneal dialysis (CAPD) is one of RRT that can be carried out. The crucial factor affecting survival rate in patients with CAPD are age and etiology of ESRD. The aim of this study is to analyze the correlation between age and etiology with survival rate on CAPD patients in Zainul Abidin General Hospital.

Methods: This study is descriptive analytic and cross-sectional survey. Data was collected from the medical record in Zainul Abidin General Hospital between 2007-2011. Twenty-eight ESRD patients treated with CAPD. The data was analyzed using chi-square and Fisher's exact test.

Results: There was no significant correlation between age and etiology with survival rate $(p=1.000 ; p=0.246)(p=0.050 .05)$. Patients in middle age, $54.5 \%$ have survival rate less a year and survival rate more than a year is $45.5 \%$. That is no different with the adult patients, the survival rate less a year and more than a year is $50 \%$. In the diabetic's patients, their survival rate in less a year is $63.64 \%$ and in nondiabetic's patients the survival rate more than a year is $58.82 \%$.

Conclusions: The middle-age patients' survival rates with diabetes mellitus less than a year and the adult with nondiabetes more than a year.

Maimun S., ${ }^{1}$ Liza S., ${ }^{2}$ Eva M.M.F. ${ }^{3}$ Division of Nephrology of Internal Medicine, ${ }^{1}$ Public Health Department, ${ }^{2}$ Syiah Kuala University Faculty of Medicine, ${ }^{3}$ Banda Aceh, Indonesia.

\section{Better Sleep Quality in Peritoneal Dialysis than Hemodialysis}

Objectives: Sleep disorders are very common in dialysis patients. Poor sleep quality is associated with poor quality of life, cardiovascular events and higher risk for death. There are few data compared the prevalence and characteristics of sleep disorders in hemodialysis (HD) patients and peritoneal dialysis (PD) patients. This study assessed sleep quality in patients on HD and PD, compare the sleep quality scores, discussed clinical factors which may impact the different prevalence of sleep disturbance in $\mathrm{HD}$ patients and $\mathrm{PD}$ patients.

Methods: A total of 53 control subjects, 112 maintenance HD patients and 91 maintenance continuous ambulatory peritoneal dialysis (CAPD) patients received questionnaire survey of Pittsburgh Sleep Quality Index (PSQI) to assess sleep quality. Demographic parameters such as gender, age, dialysis vintage were recorded; serum calcium, phosphorus, intact parathyroid hormone (iPTH), hemoglobin, albumin, lipid, Kt/N and other laboratory parameters were recorded, as well. Global PSQI $>5$ indicates poor sleeper, $\mathrm{PSQI} \leq 5$ indicates good sleeper. $\chi c^{2}$ tests, ANCOVA test were used to compare differences between groups. The impacts of clinical factors on sleep quality were analyzed by multivariate linear regression and logistic regression.

Results: Dialysis patients (HD \& PD) had higher PSQI scores than normal controls (HD, $7.38 \pm 4.70$ vs. PD, $5.78 \pm 4.00$ vs. Control, $3.28 \pm 2.79$ $p<0.05)$, and more poor sleepers than control (HD, $58.04 \%$ vs. PD, $41.76 \%$ vs. Control, $13.21 \%, p<0.05)$. Compare to HD patients, $\mathrm{PD}$ patients had lower PSQI scores and less poor sleepers $(p<0.05)$. Age $(\mathrm{OR}=1.75)$, dialysis vintage $(\mathrm{OR}=1.26)$, hemoglobin $(\mathrm{OR}=0.64)$, calcium phosphate product $(\mathrm{OR}=1.60)$ were significantly related to sleep quality score in $\mathrm{HD}$ patients $(p<0.05)$. iPTH $(O R=1.16)$ were significantly related to $P S Q$ score in PD patients $(p<0.05)$. Serum phosphorus was higher in HD patients $(H D, 1.91 \pm 0.53$ vs. $\mathrm{PD}, 1.63 \pm 0.46, p<0.05)$

Conclusions: Sleep disturbance is common in dialysis patients, while PD patients had better sleep quality than HD patients. Less hyperphosphatemia in PD patients might be one reason.

Pang H.H., Yan Y.C., Zhu M.L., Zhang W.M., Fang W., Lin A.W., Ni Z.H., Qian J.Q. Renal Division, Renji Hospital, Shanghai Jiaotong University School of Medicine, Shanghai, China.

\section{Physical Activity in Patients on Automated Peritoneal Dialysis vs. Continuous Ambulatory Peritoneal Dialysis}

Background: It is generally considered that patients on automated peritoneal dialysis (APD) can spend their free time more than those on continuous ambulatory peritoneal dialysis (CAPD). However, the differences in physical activity of patients on CAPD and those on APD have not been reported yet.

Purpose and Method: To evaluate the physical activity of CAPD and APD patients, we measured the average number of steps per day (steps/ day) of $22 \mathrm{PD}$ patients by using pedometers over one month.

Results: Twenty-two PD patients including 9 CAPD patients and $13 \mathrm{APD}$ patients were examined. They were similar in age $(62.9 \pm 7.1$ vs. $62.0 \pm 8.6$ years), sex ( $66 \%$ vs. $61 \%$ male), frequency of DM ( $33 \%$ vs. $38 \%$ diabetic), duration of dialysis ( $22.4 \pm 7.4$ vs. $18.9 \pm 18.3$ months), and BMI ( $23.8 \pm 4.6$ vs. $\left.24.0 \pm 4.2 \mathrm{~kg} / \mathrm{m}^{2}\right)$. There was also no significant difference in residual kidney function ( $24.3 \pm 13.8$ vs. $30.7 \pm 16.3 \mathrm{~L} /$ week $\left./ 1.73 \mathrm{~m}^{2}, p=0.62\right)$, quantified dose of $\mathrm{PD}(\mathrm{Kt} / \mathrm{V} 1.88 \pm 0.2$ vs. $1.79 \pm 0.3, p=0.72$ ), or blood pressure (SBP $143.7 \pm 18.5$ vs. $136.0 \pm 21.1 \mathrm{mmHg}, p=0.46$, DBP $81.7 \pm 15.8$ vs. $79.0 \pm 8.0$ $\mathrm{mmHg}, p=0.76)$. Furthermore there was no significant difference in the degree of anemia ( $\mathrm{Hb} 10.8 \pm 1.1 \mathrm{vs} .10 .1 \pm 0.9 \mathrm{~g} / \mathrm{dl}, p=0.12$ ), nutrition status (Alb $3.8 \pm 0.2$ vs. $3.7 \pm 0.3 \mathrm{~g} / \mathrm{dl}, p=0.79$ ) and inflammation (CRP $0.28 \pm 0.29$ vs. $0.32 \pm 0.55 \mathrm{mg} / \mathrm{dl}, p=0.14$ ), which could affect physical activity. The steps/ day of CAPD patients were $5286 \pm 2323$ (2126 to 8841 ), and that of APD patients were $3783 \pm 2480$ (496 to 8483$)(p=0.10)$.

Conclusion: The results showed that physical activity was not affected by the modality of peritoneal dialysis.

Shimizu S., Oishi D., Sueki S., Tsuruoka K., Kawarazaki H., Tominaga N., Sakurada T., Shirai S., Yasuda T., Kimura K Division of Nephrology and Hypertention, Department of Internal Medicine, St Marianna University School of Medicine, Kawasaki, Japan. 


\section{Rehabilitation, Quality of Life and Survival}

\section{Risk Factors for Morbidity and Mortality in Peritoneal Dialysis Patients: a Single Center Experience}

Objective: To determine the risk factors for peritoneal dialysis (PD) dropout, hospitalisations and mortality in PD patients.

Methodology: A prospective study included patients aged 18 and above who had been on PD for at least 3 months. Patients with contraindications to Body Composition Monitoring (BCM) were excluded. Baseline demographics, comorbidities, treatment information and ultrafiltration capacity were documented. Blood investigations, echocardiogram, Subjective Global Assessment (SGA) scores, anthropometric measurements, and BCM were done at baseline. Patients were followed up for 6 months and details of deaths, hospitalizations and PD dropouts were documented. Normohydration was defined as $\mathrm{OH} \leq 1.0$ $\mathrm{L}$ and overhydration as $\mathrm{OH}>1.0 \mathrm{~L}$ on $\mathrm{BCM}$.

Results: 80 patients were recruited. There were 36 hospitalisations ( 32 patients with $\mathrm{OH}>1.0 \mathrm{~L}$ vs. 4 with $\mathrm{OH} \leq 1.0 \mathrm{~L}$ ), a majority $(75 \%)$ for noninfectious causes (mainly for fluid overload and hypertensive urgencies). Patients with $\mathrm{OH}>1.0 \mathrm{~L}$ at baseline had significantly more frequent hospitalisations $(p=0.024)$ and longer hospital stay (mean 5.0 vs. 0.8 days, respectively, $p=0.034)$. Hospitalized patients also had lower serum albumin $(p=0.030)$. On follow-up, 6 patients died ( 3 acute coronary syndromes, 1 acute pulmonary oedema and 2 septicaemia) and 5 were converted to hemodialysis ( 4 persisten fluid overload and 1 recurrent peritonitis). All patients who died had $\mathrm{OH}>1.0$ $L$, with low serum albumin $(p=0.037)$ and left ventricular hypertrophy (LVH) $(p=0.017)$ at baseline. As for patients converted to $\mathrm{HD}$, they were significantly overhydrated $(p<0.001)$, had low serum albumin $(p=0.010)$, low hemoglobin $(p=0.013)$ and had poor ejection fraction $(p=0.017)$. Acidosis, anthropometric measurements and other biochemistry at baseline had no significant correlation with hospitalisation, technique and patient survival.

Conclusion: Overhydration, low serum albumin and poor cardiac function were common factors for both morbidity and mortality in PD patients. Study limitations were the short follow-up period and the small number of patients.

Sivathasan S., Mushahar L., Yusuf W.S., Ideris S., Omar S. Hospital Tuanku Ja'afar Seremban, Seremban, Malaysia.

\section{A Ten-year Audit of CAPD Outcomes from the Top End of Australia}

Aim: To assess the demography, clinical features, infection rates and outcomes of patients on continuous ambulatory peritoneal dialysis (CAPD), and to study the difference between the Indigenous and non-Indigenous Australians at the top end of Australia.

Methods: Design: Retrospective study. Inclusion: Adult CAPD patients. Period: January 2001 to December 2010. Exclusion: CAPD commenced elsewhere. Statistical analysis: SPSS v16 for Windows.

Results: 120 subjects started CAPD at the Top-end (92 Indigenous, 28 non-Indigenous). At last follow-up, 23 remained on CAPD, 52 switched to hemodialysis, 5 underwent renal transplantation, and 4 were lost to followup, while 36 died. Indigenous had higher prevalence of diabetes [ $\mathrm{n}=56$ $(60 \%)$ vs. $n=7(25 \%)$ ] and lived farther from the center [ $341.3 \mathrm{vs.} 115.9 \mathrm{Km}$ ] The cumulative peritonitis rate was 89 episodes per 100 patient-years. The infection rate was 1 episode per 13.08 months for indigenous and 1 episode per 15.30 months for non-Indigenous respectively ( $p=N S$ ). Gram-positive, gram-negative, mixed growth, fungal and culture negative peritonitis was encountered in $51.3 \%, 19.3 \%, 5.5 \%, 8.3 \%$, and $15.4 \%$, respectively. $71.1 \%$ of exit-site infections were due to gram-negative organisms. The 1 - and 2 -year technique survival was $80 \%$ and $40 \%$ while the patient survival was $85 \%$ and $72 \%$, respectively which was similar between the indigenous and non-Indigenous groups.

Conclusions: There were no significant differences between the Indigenous and non-Indigenous CAPD population other that the prevalence of diabetes and distance to the hospital. The peritonitis infection rate in Top End is quite high compared to other centers. The technique survival is also poor.

Sundaram M., Jabbar Z., Perry G., Majoni W.S., Shaw M.J. Royal Darwin Hospital, Northern Territory, Australia.

\section{Survival Comparison between High Transport and Low Transport in CAPD Patient in a Single Center}

Objective: Increased peritoneal membrane permeability is associated with higher morbidity and mortality in peritoneal dialysis patients. But it is not clear whether the difference in clinical outcome between high and low peritoneal transport groups is due to differences in peritoneal fluid and solute removal. We studied in order to compare the survival rate and causes of death in patient in CAPD between high and low peritoneal transport patterns.

Method: Standard peritoneal equilibrium test (PET) was performed in 64 CAPD patients in KangNung Asan Hospital between Jan. 2005 and Dec. 2010. Demographic and clinical characteristic findings at the beginning of CAPD were retrospectively analyzed. All subjects were followed up until the point of assessment, the time of death or withdrawal from dialysis. The survival rate was calculated by Kaplan-Meyer method.

Result: We divided into two transport groups between high (high plus high-average, $\mathrm{N}=34$ ) and low (low plus low-average, $\mathrm{N}=30$ ) peritoneal transport patterns according to their $\mathrm{D} / \mathrm{P}$ of creatinine. The mean age was $58.01 \pm 1.71$ in high group, $54.56 \pm 0.80$ in low group. Major cause of ESRD was diabetes and major cause of death was sepsis due to infection. In high transporter, patient survival rates were $83.5 \%$ at 1 year, $74.3 \%$ at 3 year and $61.2 \%$ at 5 year. In low transport, patient survival rates were $83.5 \%$ at 1 year, $74.3 \%$ at 3 year and $61.2 \%$ at 5 year. In low transport, survival rates were $80.2 \%$ at 1 year, $71.6 \%$ at 3 year and $56.6 \%$ at 5 year. The difference between high and low was not statistically significant $(p=0.67)$.

Conclusion: It is concluded that the overall patient survival was similar between high and low transport. Prospective randomized studies are needed to assign a cause-and-effect relationship between the transport characteristic and patient survival.

Song K.I., Kim S.H. Department of Internal Medicine, College of Medicine and Asan Kangnung Hospital at University of Ulsan, Kangnung, South Korea.

\section{The Clinical Effectiveness of Low Protein (LPD) and Na Restriction} (NaR) in Peritoneal Dialysis (PD)

Purpose: Severe arteriosclerosis of the CKD patients has been progressed and mostly died of cerebrocardiovascular complications. Volume control is the most important in the patient management; therefore, we examined the effectiveness of LPD and $\mathrm{NaR}$ in PD.

Methods and Subjects: We maintain 167 chronic haemodialysis (HD) cases (mean age $68 \pm 12$ years, mean HD period $80 \pm 50$ months, 50 PD cases (mean age $65 \pm 10$ years, mean PD period $33 \pm 33$ months). There were 75 PD patients, mean age $63 \pm 13$ years, who had been newly induced to $\mathrm{PD}$ for about ten years. We have been keeping the nutritional education and LPD $(0.8 \mathrm{~g} / \mathrm{kgBW})$ and $\mathrm{NaR}(5-6 \mathrm{~g} /$ day $)$ by the renal dietician. We analyzed for the data of blood examination, blood pressure, body fluid status, the patient survival, and cardiac function.

Results: In our department there was $62.3 \%$ of 5-year PD therapy continuation, $78 \%$ of 5 -year patient survival rate in all PD patients. Meanwhile, about ten years, there were $6 \mathrm{PD}$ cases on the number of the death. The cause of death was firstly, infection $67 \%$, malignant tumor $17 \%$, and no cases died of myocardial infarction or strokes in PD. There are two patients undergoing $\mathrm{PD}$ for over ten years. Also their peritoneal equilibrium tests (PET) are still low-average. About $83.2 \%$ of 19 long-term PD patients (LCAPD), their consecutive PET data of PD do not worsen for 4 years. The PD cases who can keep LPD $(0.8 \mathrm{~g} / \mathrm{kg})$ will maintain the PET data. The data of blood pressure were $119 \pm 69 \mathrm{mmHg}$. Echocardiographic findings showed that left ventricular mass index (LVMI) of PD patients was almost equal $107 \pm 6 \mathrm{~g} / \mathrm{cm}^{2}$ compared to those $113 \pm 4$ of HD. There was no significant difference between the two groups (HD and LCAPD) in clinical data.

Conclusions: LPD and NaR in PD could reduce the risk of peritoneal hyperpermeability, and might improve cardiovascular risk of PD equal to those of HD patients ( 15 hours a week).

Takeda K., Mukai H. Dept of Kidney Center, and Nephrology, Aso-lizuka Hospital, lizuka, Japan. 


\section{Rehabilitation, Quality of Life and Survival}

\section{Clinical Outcomes and Mortality in Peritoneal Dialysis Patients: a Ten-year Retrospective Analysis in a Single Center}

Objective: To evaluate the clinical outcome, to identify predictors of patients and technical survival in PD patients in the west region of Turkey.

Methods: We included patients who initiated therapy between 2001-2010. Sociodemographic characteristics were investigated from files. HD history and duration, additional systemic diseases, and ESRD etiologies of patients were recorded. Clinical data and laboratory parameters were evaluated pretreatment and during the last monitoring period. Infectious complications and their incidences were searched. Patient and technique survival were investigated for every patient.

Results: 342 patients (pts) were started PD treatment. 43patients were excluded. Data of remaining 299 patients ( $167 \mathrm{~F}$, mean follow-up $38.5 \pm 26.8$ months mean age $44.7 \pm 15.9$ years) were evaluated retrospectively. It was determined that $87.3 \%$ of the patients made their PD exchanges without help from anyone. Preference of PD - done by themselves in $79.9 \%$ patients. Peritonitis incidences and catheter exit-site/tunnel infection attacks were as $27 \pm 23$ and $32.3 \pm 24.9$ patient-months, respectively. During the follow-up period 199 patients $(80$ transfer to $H D, 78$ death, 41 transplantation) were withdrawn from PD. The most frequent causes of death were cardiovascular events and peritonitis and/or sepsis. Whereas transfer to $\mathrm{HD}$ was due to peritonitis and/or sepsis. Mean patients survival time were 49.9 \pm 2.6 months. Preference of PD (RR:4.77, $p<0.001$ ), HD history (RR:2.08, $p=0.04$ ), diabetic status (RR:2.13, $p=0.01$ ), pretreatment albumin (RR:0.32, $p<0.001$ ) and the last parathormon levels (RR:0.99, $p=0.04$ ) predicted mortality. Mean technique survival duration were $48.5 \pm 2.4$ months. Technique survival was associated with preference of PD (RR:0.45, $p<0.001$ ), diabetic status (RR:1.92, $p=0.003$ ) and pretreatment albumin levels (RR:0.58, $p=0.003$ ).

Conclusion: Patient survival in the presented institute is similar to that reported in Western countries. Compulsory choice of preference PD, HD history, diabetic status, low pretreatment serum albumin and the last low serum parathormon levels were the strongest predictors of death. Risk factors of technique failure were compulsory choice of preference PD, diabetic status, and low pretreatmen serum albumin

Unsal A., Koc Y., Basturk T., Ahbap E., Sakaci T., Sinangil Arar A. Department of Nephrology, Sisli Etfal Research and Educational Hospital, Istanbul, Turkey.

\section{Effects on Survival of Etiological Causes in Peritoneal Dialysis}

Objective: to investigate the effect on mortality in ESRD etiologies in PD patients (pts).

Methods: We included patients who initiated therapy between 2001-2011. Patients were divided into four groups regarding to etiologies.Group1, 2, 3 and 4 were consisted of amyloidosis, diabetes mellitus, chronic glomerulonephritis and polycistic renal disease, respectively. Sociodemographic data, clinical courses and the infectious complications of the between groups were compared, and the reasons for PD withdrawal were obtained. Patient and technique survival analysis of patients were performed.

Results: 354 pts were started PD treatment, 154 pts were excluded. Data of remaining 204 patients were evaluated. A total of $29 \mathrm{AA}$ amyloidisis $(14 \mathrm{~F}$, mean age $37.9 \pm 16.4$ years, mean follow-up time $21.7 \pm 20.2$ months) were in group 1,78 diabetes mellitus ( $41 \mathrm{~F}$, mean age $56.9 \pm 13.6$ years, mean follow-up time $35 \pm 28.6$ months) were in group 2, 68 chronic glomerulonephritis ( $44 F$, mean age $37.2 \pm 12$ years, mean follow-up time $47.7 \pm 29.9$ months) consist of group 3 , and 29 polycystic renal disease $(12 \mathrm{~F}$, mean age $35.6 \pm 13.8$ years, mean follow-up time $45.4 \pm 36.8$ months) were in group 4 . There was no difference between groups in terms of educational status $(p=0.055)$. The albumin level was lower in group 1 at initial and the last visit of PD (for both $p<0.001$ ). Peritonitis incidences and catheter exit-site/ tunnel infection attacks were higher in amiloidotic patients $(p=0.002$ and 0.018 , respectively). There was a difference between groups with respect to the last status of patients $(p<0.001)$. Group1 and 2 had more patients die. The major causes of deaths were peritonitis and/or sepsis and cardiovascular reasons in all patients. The mortality rate was found higher in amyloidotic patients (log rank=0.005), especially first 2-3 years. Who helped administer the PD (OR:6.244, $p=0,025)$, initial serum albumin (OR:0.352, $p=0,034)$. and presence of catheter exit-site/tunnel infection (OR:0.250, $p=0,015$ ) were found to predict patient survival. The technique survival rate was similar found between groups (log rank $=0.238$ ).

Conclusion: Causes of etiologies have to effects on PD patient survival, amyloidosis patients has the worst survival. In amyloidotic patients, PD may not be considered as first choice therapy.

Koc Y., Basturk T., Unsal A., Sakaci T., Ahbap A., Sinangil Arar A., Doner B., Akgol C. Department of Nephrology, Sisli Etfal Research and Educational Hospital, Istanbul, Turkey.

\section{Factors Associated with Five-year Survival in Peritoneal Dialysis Patients}

Aim: We investigated 5 years more and under patient survival and factors affecting mortality in peritoneal dialysis (PD) patients.

Methods: This was a retrospective study including 354PD patients. The demographic, clinical, and biochemical data were collected from the medical records.200 patients were excluded. 154 patients' data were evaluated, including 83 patients surviving 5 years or more and 71 patients those were taken as controls (nonsurvivors).

Results: Mean age $(p=0.001)$, number of comorbid diseases $(p=0,001)$, prevalance of $\mathrm{DM}(p=0.002)$, mandatory preference of $\mathrm{PD}(p<0.001)$, making their $\mathrm{PD}$ exchanges with help from anyone $(p<0.001)$ were lower and education level were higher $(p=0.041)$ in surviving patients compared nonsurvival group. At the beginning serum creatinine $(p=0.011)$, albumin $(p=0.001)$ and $\operatorname{RRF}(p=0.017)$ were all higher in the survival group than in the nonsurvival group. In addition, peritoneal permeability differed between the groups also $(p=0.006)$. Incidence of peritonitis and catheter exit-site/tunnel infection attacks were lower in survival group than in nonsurvival group ( $p=0.001, p=0.001$, respectively). In nonsurvival group, infectious events $(49.3 \%$ ) were the most important causes of death. Advanced age (RR:1.057, $p=0.021)$, mandatory preference of $\mathrm{PD}$ (RR:0.999, $p=0.049)$, high baseline high and high-average PET transporters rates (RR:0.181, $p=0.008$ ), low albumin level ( $R R=0.333, p=0.002$ ), high peritonitis status ( $R R=0.056, p=0.001$ ), and decreased $R R F(R R=1.006, p=0.003$ ) were associated with an increased risk of death. In survival group, most common cause of death is $\mathrm{CVH}(47.6 \%)$. Self preference of $\mathrm{PD}(\mathrm{RR}=0.001, p=0.004)$, high baseline low, low-average PET transporters rates ( $R R=0.009, p=0.001)$ and initia RRF (RR:0.985, $p=0.013$ ) were factors effective on mortality.

Conclusion: Long-term patients' survival is possible for PD patients, particularly in ones with younger age, nondiabetics, having higher education level, lowe number of comorbid disease, self preference of PD, making PD exchanges without any help, preserving residual renal function, having low peritonitis rate and low and low-average PET transporters. The most common cause of death was infections in nonsurviving group, while it was cardiovascular-related problems in surviving group. Even though the most common reasons of death were different, there were no differences between groups $(p>0.05)$.

Unsal A., Basturk T., Koc Y., Sinangil Arar A., Ahbap E., Sakaci T., Kara E., Kayalar A. Department of Nephrology, Sisli Etfal Research and Educational Hospital, Istanbul, Turkey.

\section{Is There Effect of Body Mass Index on Survival in Peritoneal} Dialysis Patients?

Objective: We examined the effect of higher than normal BMI on patient and technique survival in PD patients to determine the main risk factors affecting survival.

Methods: A total of 354 patients treated with PD between 2001-2011 were evaluated retrospectively. 138 patients were excluded. Data of remaining 216 patients ( $100 \mathrm{~F}$, mean follow-up months $42.4 \pm 31$, mean age $44.8 \pm 15$ years) were evaluated retrospectively. Patients were separated into two groups as group 1 ( $\mathrm{n}: 130)$; normal BMl (18.5-24.9 kg/m²) and group 2 (n:86); highBMl (>25 kg/m²). Blood sample and baseline characteristics of the study cohort were obtained at the start of PD. Patient and technique survival were compared using Cox proportional hazards model.

Results: There were statistical differences in age $(41.7 \pm 15.6 / 51.6 \pm 11.4$ years, $p=0.001)$ and presence of diabetes $(19.5 \% / 36.2 \%, p=0.015)$ other parameters (gender, mean follow-up, initial $\mathrm{Kt} / \mathrm{V}, \mathrm{CCID} / \mathrm{PCr}$, and serum albumin levels showed no differences between groups ( $p>0.05$ for all). Mean patient survival was $69.4 \pm 5.2$ and $67.7 \pm 5.3$ patient-months in two groups, respectively. Kaplan-Meier survival analysis showed similar 1, 3, 5-year patient survival two groups $(92,78.9,60.4 \%$ vs. $88.8,85.8,62.1 \%$ ). Kaplan-Meier survival curve revealed that PD patients who were group 1 do not have a worse survival compared with group 2 (log-rank: 0.89). Cox proportional hazards analysis revealed that $\mathrm{D} / \mathrm{PCr}(\mathrm{RR}: 0.134, p=0.006)$, initial albumin level (RR:0.343, $p=0.001$ ), presence of diabetes (RR:0.313, $p=0.002$ ) and RRF (RR:0.999, $p=0.012$ ) were risk factors associated with all-cause mortality. Mean technique survival were found $45 \pm 3.6$ and $55.4 \pm 5.5$ patient-months in two groups, respectively. Technique survival was also similar between the two groups $(84.9,51.8,27.5 \%$ vs. 84.5, 58.4, 43.8\%) (log-rank: 0.12). With Cox proportional hazards analysis revealed, age (RR: $0.970, p<0.001$ ), peritonitis status (RR:3,124, $p<0.001)$ catheter exit-site/tunnel infection status (RR:1.790, $p=0.004)$ and initial serum albumin level (RR:1,637, $p=0.018$ )

Conclusions: $\mathrm{PD}$ patients who are high BMl at the start of dialysis have similar survival compared with PD patients with a normal BMI. Presence of diabetes, baseline peritoneal.

Basturk T., Koc Y., Unsal A., Ahbap E., Sinangil Arar A., Sakaci T., Sevinc M., Atanucar Z. Department of Nephrology, Sisli Etfal Research and Educational Hospital, Istanbul, Turkey. 


\section{Rehabilitation, Quality of Life and Survival}

\section{Outcome of Patients Started on PD as First-line Therapy Compared to Patients Transferred from Hemodialysis}

Objectives: To assess complications and outcome of patients started on peritoneal dialysis $(\mathrm{PD})$ as initial dialysis therapy, compared to PD after hemodialysis (HD).

Methods: We included 125 patients in PD unit during a 10 year period, (2002-2011). 86 patients started on PD as initial therapy, and 39 patients started PD after regular HD. We compared demographic and biochemical data, outcome, and complications.

Results: In PD group, 18 (20.93\%) patients were on continuous ambulatory peritoneal dialysis (CAPD), while $68(79.11 \%)$ on automated peritoneal dialysis (APD). Mean age was $43.84(14-82 \pm 19.314)$ years. Mean time on PD was $49.37(3-168 \pm 41.43)$ months. The mortality rate was $13.9 \%$ (12 patients). At end of study, 19 patients $(22.1 \%$ ) had been transferred to $\mathrm{HD}, 14(16.27 \%)$ underwent renal transplant, $4(4.65 \%)$ transferred to another hospital, and $36(41.86 \%)$ were continuing on PD. The peritonitis rate was 1 episode every 51.94 patient months. In the HD group, 7 patients (17.9\%) were on CAPD, while $32(82.1 \%)$ on APD. The mean age was $62.23(21-86 \pm 16.153)$ years. The mean time on PD was 29.95 (3-92 \pm 22.9 ) months. Mortality rate was $25.64 \%$ (10 patients). At end of study, 21 patients (35.9\%) transferred back to HD, $2(5.1 \%)$ underwent renal transplant, while $4(10.25 \%)$ transferred to another hospital. 9 patients (23.08\%) were continuing on PD at end of study. The peritonitis rate was 1 episode every 33.78 patient months.

Conclusion: Over a ten-year period, patients started on renal replacement therapy with PD showed a better patient and technique survival compared to patients initially on HD. The patients in the PD group showed longer time on $\mathrm{PD}(p=0.008)$, lower mortality $(p=0.01)$ and fewer peritonitis episodes $(p=0.027))$. However, this can be attributed to differences in age and sample size.

Usama S., Alwakeel J.S., Mitwalli A.H., Alsuwaida A., Askar A. King Khalid University Hospital, Saudi Arabia..

\section{Patient and Technique Survival in Peritoneal Dialysis} Patients with Prior Stroke

Objectives: The purpose of this study was to investigate patient survival, death-censored technique failure and peritonitis-free survival in continuous ambulatory peritoneal dialysis (CAPD) patients with prior stroke.

Methods: This is a retrospective observational cohort study. CAPD patients from PD center of The First Affiliated Hospital, Sun Yat-sen University were enrolled from 1 January 2006 to 31 December 2010. All patients were divided into stroke group and nonstroke group on the basis of prior stroke presence. The primary outcome was patient survival. Survival analysis was used by the Kaplan-Meier method and survival curves comparison by the Mantel-Haenszel log-rank test.

Results: A total of 1144 patients (age $48.30 \pm 15.80$ years) were enrolled, including $89(7.7 \%)$ patients in the stroke group (mean age $63.1 \pm 10.5$ years) and $1055(92.3 \%$ ) patients in the nonstroke group (mean age $47.1 \pm 15.5$ years). Duration of follow-up was $28.03 \pm 15.25$ months. A total of 165 patients died during the follow-up, including 29 in the stroke group and 136 in the nonstroke group. Patient survival, technique survival and peritonitis-free survival were significantly lower in the stroke group compared with that in nonstroke group (Log rank $=24.21, p<0.001$; Log rank $=6.09, p=0.014$ and Log rank $=9.58, p=0.002$, respectively). Additionally, time to first peritonitis was the independent predictor for patient survival and death-censored technique failure in the stroke group (HR $0.9395 \% \mathrm{Cl}$ $0.88-0.98, p=0.003$ and $\mathrm{HR} 0.9395 \% \mathrm{Cl} 0.87-0.99, p=0.028$, respectively).

Conclusions: CAPD patients with prior stroke had poor patient and technique survival, as well as peritonitis-free survival compared to that in the nonstroke group. Additionally, time to first peritonitis was an independent predictor for patient and technique survival in these patients.

Wu X., Yang X., Feng X., Yi C., Yu X. Department of Nephrology, The First Affiliated Hospital, Sun Yat-sen University, Guangzhou, China.

\section{A Prospective, Randomized, Multicenter Study Comparing Survival in Subjects Receiving Peritoneal Dialysis or Hemodialysis (SURinD): Feasibility and Preliminary Enrollment Data}

Objectives: There is no consensus on whether peritoneal dialysis (PD) or hemodialysis (HD) is associated with better survival outcomes in patients with end-stage renal disease (ESRD). To date, no large randomized study has compared survival in patients receiving $\mathrm{PD}$ or $\mathrm{HD}$ because of difficulties recruiting and randomizing patients. However, in a pilot study conducted in China, we showed that $61 \%$ of eligible subjects were willing to be randomized to either dialysis modality, indicating that an adequately powered, randomized study is feasible.

Methods: SURinD is a prospective, randomized, multicenter, open-label, noninferiority study being conducted at 30 sites (expected) in China (NCT01413074 Baxter Healthcare). The primary objective is to compare survival in subjects receiving $\mathrm{PD}$ or $\mathrm{HD}$ (assessed by $\mathrm{PD} / \mathrm{HD}$ mortality hazard ratio). Secondary objectives include comparisons of technique failure, cause of death, residual renal function, erythropoietin-stimulating agent dose, systemic inflammation, quality of life, and safety. Approximately 1,370 subjects ( $\geq 18$ years, diagnosed with ESRD [glomerular filtration rate $\leq 15 \mathrm{~mL} / \mathrm{min} / 1.73 \mathrm{~m} 2$ body surface area], predicted to need dialysis within 10 weeks) will be randomized (centralized randomization, stratified by investigator site) to $\mathrm{PD}$ or $\mathrm{HD}(1: 1)$ and followed for up to 5 years. Sample size is based on an overall type I error of $5 \%$, a power of $80 \%$, and a noninferiority margin of 1.25 for the PD/HD mortality hazard ratio. Assessments will ensure that internationally accepted standards of care and clinical practice guidelines for dialysis are met for both modalities. Trial governance includes an independent, international data and safety monitoring board and an international advisory board.

Results: Recruitment began in September 2011; by February 2012, 69 subjects had been enrolled at 9 study sites. Study completion is expected in 2016

Conclusions: SURinD will be the first large randomized study to compare survival outcomes in patients with ESRD receiving PD or HD.

Yu X., ${ }^{1}$ Qian J., ${ }^{2}$ Shi Y., ${ }^{3}$ Chen H. ${ }^{3}$ Zuo L., ${ }^{3}$ Jiang A., ${ }^{3}$ Yang H., ${ }^{3}$ Chen N., ${ }^{3}$ Hao C..${ }^{3}$ Tranaeus A. Vonesh E.F ${ }^{3}$ Mao $\mathrm{J}^{3}$ Yao $\mathrm{O}^{3}$ Dept of Nephrol, ${ }^{1}$ The First Affiliated Hosp, Sun Yat-sen Univer Guangzhou, China; Renal Div, ${ }^{2}$ Renji Hosp, Shanghai Jiao-Tong Univer School of Med, Shanghai, China; SURinD Research Group, ${ }^{3}$ Shanghai,China.

\section{The Effects of Joint Follow-up Model on the Outcome of Peritoneal Dialysis Patients Lived in Remote Areas}

Objective: Since more than $65 \%$ peritoneal dialysis $(\mathrm{PD})$ patients in our PD center lived far away from our city, a joint follow-up model was established, by which our PD center combined with satellite PD centers or other local PD centers are available for these PD patients. The aim was to investigate the effects of this model on the outcome of PD patients lived in remote areas.

Methods: 521 PD patients who lived in remote areas and commenced PD treatment more than 90 days from January 2006 to December 2009 were recruited. All patients were divided into 3 groups: group $A$ (followed up in joint follow-up model every 1 6 months, $n=198$ ), group $B$ (followed up only in our PD center every 1 6 months, $n=214$ ), group C (irregular follow-up, $n=109$ ). Demographic characteristics, laboratory parameters at the time of dialysis initiation and clinic events during the study were collected. Kaplan-Meier analysis and Cox regression analysis was used to assess predictors of survival.

Results: Of all patients, the median age was 46.0 (33.0 58.0) years, the median vintage of PD was 22.5 (14.7 34.1) months, and the primary renal disease was glomerulonephritis $(55.9 \%)$, followed by diabetic nephropathy $(19.6 \%)$. The vintage of PD in group A was longer than that in group B and group $C$ (all $p<0.01$ ). Self-payment proportion in group $A$ was comparable to group $B$ and both were lower than that in group $C$ (all $p<0.01$ ). Patient survival in group $A$ was comparable to group $B$ and both were better than group $C$ (all $p<0.01)$. Irregular follow-up and older age were predictors of mortality in PD patients in remote areas (HR: 2.447, 1.030; all $p<0.05$ ). Technique survival was not significantly different among three groups. Self-payment (HR: 2.912; $p<0.05$ ) was predictor of $P D$ technique failure in PD patients in remote areas.

Conclusions: The outcome of patients followed up by a joint model was comparable to the regular main PD center visit model. The joint follow-up model could benefit the PD patients who lived in remote areas, and improve their patient survival.

Yi C., Yang X., Guo Q., Jiang Z., Lin J., Mao H., Yu X. Department of Nephrology, The First Affiliated Hospital, Sun Yat-sen University, Guangzhou, China. 


\section{Rehabilitation, Quality of Life and Survival}

\section{Peritoneal Dialysis in the Treatment of Focal Segmental} Glomerulosclerosis Patients with Acute Kidney Injury

Objective: To observe the efficacy and safety of peritoneal dialysis (PD) in the treatment of focal segmental glomerulosclerosis (FSGS) patients with acute kidney injury (AKI).

Methodology: Nineteen FSGS patients with AKI were enrolled in this study. They were received DAPD with a daily exchange of $1 \sim 8 \mathrm{~L}$ for at least 4 months. The clinical efficacy, peritoneal dialysis related complications, and biochemical parameters were reviewed.

Results: The patients had a mean follow-up of more than 3 months. The clinical efficacy rare was $73.7 \% .10(52.6 \%)$ of the 19 cases had a normal renal function, 4 cases got rid of dialysis, 4 cases still were received peritoneal dialysis treatment, and 1 switched to hemodialysis therapy. More than $70 \%$ of patients had safety volume and blood pressure control. At the end of follow-up, the level of serum creatinine was decreased to $65.6 \%$ of baseline $(p>0.05)$, and BUN was decreased to $47.9 \%$ of baseline $(p<0.01)$. The patients also had increased serum albumin, pre-albumin and urine output ( $p>0.01)$, and decreased proteinuria, urine NAG and RBP $(p<0.01)$. Edema usually subsided within 4 weeks of PD therapy and the time of renal function recovery was 1 to 6 months. The PD-related complications occurred in 8 cases (42\%), including 5 peritonitis, which had appeared in $(3.13 \pm 1.44)$ month, one catheter exit infection, one poor dialysis drainage, and one dialysate leakage to the thoracic cavity. No clinically significant complications such as perivascular effusion and abdominal organ damage were noted.

Conclution: Due to its efficacy and less adverse events, short-term PD can be used as an effective auxiliary treatment for nephritic syndrome (NS) with AKI, especially for those who presented with severe edema and massive ascites, and for those who had poor response to diuretics.

Chen Y.W., Yu Y.S., Zhou Y., Li S.J., Wang H., Zhou T.T., Liu Z.H. Research Institutes of Nephrology, Jingling Hospital, Nanjing University School of Medicine, Nanjing, China.

\section{Improving Long-term Outcome of Peritoneal Dialysis:} a Cohort Study

Objectives: We compared patient characteristics and outcomes of patients initiating peritoneal dialysis (PD) in two different periods to determine secular trends and predictors of patient outcomes.

Methods: This study included all end-stage renal disease (ESRD) patients who started on PD between 1 January 2000 and 31 December 2009. They were followed up from the date of PD initiation until death, cessation of PD, transfer to other centers or to the end of the study (31 December 2010).

Results: A total of 598 patients were included, 259 started PD during 2000 to 2004 , and 339 during 2005 to 2009 period. Compared to the patients from 2000-2004 cohort, patients from 2005-2009 cohort had more instances of diabetes comorbidity ( $23.3 \%$ vs. $15.8 \%, p=0.028)$, higher haemoglobin $(104.3 \pm 22.6$ vs. $94.4 \pm 20.7 \mathrm{~g} / \mathrm{l}, p<0.001)$, lower phosphate $(1.44 \pm 0.47 \mathrm{vs}$. $1.55 \pm 0.60 \mathrm{mmo} / /, p=0.024)$, similar serum albumin ( $34.5 \pm 5.2 \mathrm{vs} .34 .5 \pm 5.8$ $\mathrm{g} / \mathrm{l}, p>0.05)$ and residual renal functions (RRF) $(3.80 \pm 2.37 \mathrm{vs} .3 .55 \pm 2.57$ $\mathrm{ml} / \mathrm{min}, p>0.05$ ) at the time of PD initiation. The patients survival rates were: $90 \%, 79 \%, 72 \%$ and $66 \%$ for $2000-2004$ cohort and $94 \%, 89 \%, 83 \%$ and $74 \%$ for 2005-2009 cohort at 1,2, 3 and 5 years, respectively. Patient survival was significant higher in patients who started PD during 2005 to 2009 (Log rank 5.547, $p=0.019$ ). Cardiovascular death remained the most common cause of death in both cohorts and patients in 2005-2009 cohort had more deaths cause by CVD ( $58.3 \%$ vs. $40.6 \%, p=0.031)$. Multivariate Cox proportional hazards modeling showed that older age, CVD, lower serum albumin were independent predictors of increased mortality in the combined cohort. There is no significant difference in technical survival between two cohorts.

Conclusions: Patient characteristics and outcomes differed between two cohorts. Our study suggests that there has been considerable improvement in long-term patient survival in patients on PD.

Zhang L., Fang W., Liu Y., Lin A., Ni Z., Qian J. Renji Hospital, Shanghai Jiao Tong University School of Medicine, Shanghai, China. 